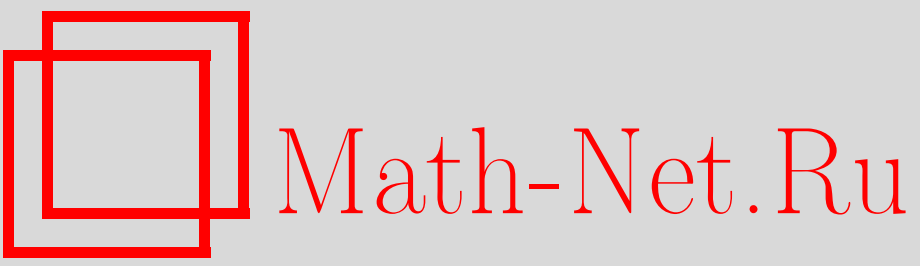

А. А. Должковой, Н. Н. Попов, Решение нелинейной стохастической задачи ползучести для толстостенной трубы методом малого параметра, Вестн. Сам. гос. техн. ун-та. Сер. Физ.-мат. науки, 2002, выпуск 16, 84-89

DOI: https://doi.org/10.14498/vsgtu102

Использование Общероссийского математического портала Math-Net.Ru подразумевает, что вы прочитали и согласны с пользовательским соглашением

http://www.mathnet.ru/rus/agreement

Параметры загрузки:

IP: 34.229 .45 .116

26 апреля 2023 г., 12:38:08 


\title{
РЕШЕНИЕ НЕЛИНЕЙНОЙ СТОХАСТИЧЕСКОЙ ЗАДАЧИ ПОЛЗУЧЕСТИ ДЛЯ ТОЛСТОСТЕННОЙ ТРУБЫ МЕТОДОМ МАЛОГО ПАРАМЕТРА
}

\begin{abstract}
Методом малого параметра во втором приближении получено решение задачи о ползучести толстостенной трубы, находящейся под действием внутреннего давления. Стохастичность введена в определяющее соотношение ползучести, взятого в виде нелинейной теории вязкого течения, при помощи случайной функции одной переменной. Вычислены дисперсии случайных скоростей деформаџий ползучести при условии, что функция, задающая случайное поле возмущений механических свойств материала, распределена по нормальному закону. Произведено сравнение результатов, полученных в данной работе, с результатами для аналогичной задачи, решенной в первом приближении.
\end{abstract}

Исследование напряженно - деформированного состояния элементов конструкций, работающих в условиях нелинейной ползучести, является достаточно сложной задачей. Сложность обусловлена скорее не поиском характеристик некоторой “осредненной” конструкции, а необходимостью учета стохастических неоднородностей материала, поскольку опытные данные по деформации ползучести, как известно, имеют значительный разброс.

В данной работе решается статистически нелинейная задача о ползучести толстостенной трубы под действием внутреннего давления $q$. Рассмотрим данную задачу в цилиндрических координатах, в предположении, что стохастические неоднородности материала оболочки трубы описываются функцией одной переменной (радиуса r). При этом компоненты тензора деформаций и тензора напряжений будут также случайными функциями только радиуса $r$.

В соответствии с теорией вязкого течения деформации ползучести $\varepsilon_{\mathrm{r}}$ и $\varepsilon_{\varphi}$ описываются следующими реологическими соотношениями в стохастической форме [1]:

$$
\begin{aligned}
& \dot{\varepsilon}_{r}=c S^{n-1} \overline{\sigma_{r}}[1+\alpha U(r)] ; \\
& \dot{\varepsilon}_{\varphi}=c S^{n-1} \frac{\overline{\sigma_{\varphi}}}{\sigma_{\varphi}}[1+\alpha U(r)],
\end{aligned}
$$

где $S$-интенсивность и $\overline{\sigma_{r}}, \overline{\sigma_{\varphi}}$ - девиаторы напряжений, которые имеют вид

$$
S=\sqrt{3} \overline{\sigma_{\varphi}} ; \overline{\sigma_{r}}=-\frac{1}{2}\left(\sigma_{\varphi}-\sigma_{r}\right) ; \overline{\sigma_{\varphi}}=\frac{1}{2}\left(\sigma_{\varphi}-\sigma_{r}\right) ;
$$

$U(r)$ - случайная функция, описывающая стохастическую неоднородность материала оболочки, характеристики которой известны: $\left\langle\mathrm{U}>=0,\left\langle\mathrm{U}^{2}>=1 ; \alpha-\right.\right.$ коэффициент вариации механических свойств; $c, n$ - постоянные материала; $<>$ - символ математического ожидания.

Напряжения $\sigma_{r}$ и $\sigma_{\varphi}$ удовлетворяют дифференциальному уравнению равновесия

$$
\frac{d \sigma_{r}}{d r}+\frac{\sigma_{r}-\sigma_{\varphi}}{r}=0
$$

и граничным условиям

$$
\sigma_{r}(a)=-q, \quad \sigma_{\varphi}(b)=0,
$$

где $a$ и $b$ - внутренний и наружный радиусы трубы.

Компоненты тензора скоростей деформаций удовлетворяют условию совместности

$$
r \frac{d \dot{\varepsilon}_{\varphi}}{d r}+\dot{\varepsilon}_{\varphi}-\dot{\varepsilon}_{r}=0
$$

Рассмотрим задачу определения напряженного состояния трубы, которая сводится к решению системы уравнений (1), (3), (5) относительно напряжений при граничных условиях (4).

Подставляя определяющие соотношения (1) в условия совместности деформаций (5), получаем уравнение (штрихом обозначается дифференцирование по r):

$$
(n-1) r S^{\prime} \overline{\sigma_{\varphi}}[1+\alpha U(r)]+r S \overline{\sigma_{\varphi}^{\prime}}[1+\alpha U(r)]+r S \overline{\sigma_{\varphi}} \alpha U^{\prime}+2 S \overline{\sigma_{\varphi}}[1+\alpha U(r)]=0,
$$

которое с использованием (2) можно привести к виду:

$$
n r\left(\sigma_{\varphi}^{\prime}-\sigma_{r}^{\prime}\right)[1+\alpha U(r)]+\alpha r\left(\sigma_{\varphi}-\sigma_{r}\right) U^{\prime}(r)+2\left(\sigma_{\varphi}-\sigma_{r}\right)[1+\alpha U(r)]=0 .
$$

Теперь, используя вытекающее из (3) соотношение

$$
\sigma_{\varphi}-\sigma_{r}=r \sigma_{r}^{\prime}
$$


уравнение (6) можно привести к виду

$$
\mathrm{n}\left(\sigma_{\varphi}^{\prime}-\sigma_{r}^{\prime}\right)[1+\alpha \mathrm{U}(\mathrm{r})]+\alpha \mathrm{r} \sigma_{r}^{\prime} \mathrm{U}^{\prime}+2 \sigma_{r}^{\prime}[1+\alpha \mathrm{U}(\mathrm{r})]=0 .
$$

Из последнего уравнения выражаем $\sigma_{\varphi}^{\prime}$ :

$$
\sigma_{\varphi}^{\prime}=\frac{\sigma_{r}\left([1+\alpha U(r)](n-2)-r \alpha U^{\prime}\right)}{n[1+\alpha U(r)]}
$$

и подставляем в уравнение

$$
\sigma_{\varphi}^{\prime}-\sigma_{r}^{\prime}=r \sigma_{r}^{\prime \prime}
$$

которое получено дифференцированием уравнения равновесия (3) по $r$. Тогда получим статистически нелинейное уравнение второго порядка относительно радиального напряжения:

$$
r[1+\alpha U(r)] \sigma_{r}^{\prime \prime}+\frac{n+2}{n}[1+\alpha U(r)] \sigma_{r}^{\prime}+\frac{r}{n} \alpha U^{\prime} \sigma_{r}^{\prime}=0 .
$$

Для приближенного решения (9) используется метод разложения напряжения $\sigma_{\mathrm{r}}$ по малому параметру $\alpha$ :

$$
\sigma_{r}=\sigma_{r 0}+\sum_{k=1}^{\infty} \alpha^{k} \sigma_{r k} \quad,\left\langle\sigma_{r}\right\rangle=\sigma_{r 0} .
$$

Подставляя (10) в уравнение (9), можно получить

$$
\begin{gathered}
r[1+\alpha U(r)]\left[\sigma_{r 0}^{\prime \prime}+\alpha \sigma_{r 1}^{\prime \prime}+\alpha^{2} \sigma_{r 2}^{\prime \prime}+\ldots\right]+\frac{n+2}{n}[1+\alpha U(r)]\left[\sigma_{r 0}^{\prime}+\alpha \sigma_{r 1}^{\prime}+\alpha^{2} \sigma_{r 2}^{\prime}+\ldots\right]+ \\
+\frac{r}{n} \alpha U^{\prime}\left[\sigma_{r 0}^{\prime}+\alpha \sigma_{r 1}^{\prime}+\alpha^{2} \sigma_{r 2}^{\prime}+\ldots\right]=0 .
\end{gathered}
$$

Уравнение (11) эквивалентно системе уравнений, полученной приравниванием множителей при одинаковых степенях $\alpha$ :

$$
\begin{gathered}
r \sigma_{r 0}^{\prime \prime}+\frac{n+2}{n} \sigma_{r 0}^{\prime}=0 ; \\
r \sigma_{r 1}^{\prime \prime}+\frac{n+2}{n} \sigma_{r 1}^{\prime}=-\frac{r}{n} U^{\prime} \sigma_{r 0}^{\prime} ; \\
r \sigma_{r k}^{\prime \prime}+\frac{n+2}{n} \sigma_{r k}^{\prime}=-\frac{r}{n} U^{\prime}\left[\sigma_{r k-1}^{\prime}-U \sigma_{r k-2}^{\prime}+U^{2} \sigma_{r k-3}^{\prime}-\ldots+(-1)^{k-1} U^{k-1} \sigma_{r 0}^{\prime}\right], \quad k=2,3,4 \ldots
\end{gathered}
$$

Решение системы (12)-(14) в рекуррентной форме связано с большими трудностями. Поэтому ограничимся системой трех первых уравнений, которую можно получить из (11) при учете только членов, содержащих $\alpha^{0}, \alpha^{1}, \alpha^{2}$, пренебрегая членами, содержащими $\alpha^{3}$. Эта система будет состоять из уравнений (12),(13) и уравнения (15), полученного из (14) при $k=2$ :

$$
r \sigma_{r 2}^{\prime \prime}+\frac{n+2}{n} \sigma_{r 2}^{\prime}=-\frac{r}{n} U^{\prime}\left[\sigma_{r 1}^{\prime}-U \sigma_{r 0}^{\prime}\right] .
$$

Уравнение (12) при граничных условиях (4) дает известное детерминированное решение [2]:

$$
\sigma_{r 0}=A\left[1-(b / r)^{2 / n}\right] \text {, где } A=\frac{q a^{2 / n}}{b^{2 / n}-a^{2 / n}} .
$$

Подставляя детерминированное решение $\sigma_{\mathrm{r} 0}$ в уравнение (13), получаем соотношение

$$
\frac{d}{d r}\left(r^{1+2 / n} \sigma_{r 1}^{\prime}\right)=-\frac{2 A b^{2 / n}}{n^{2}} U^{\prime},
$$

интегрируя которое, находим решение второго уравнения системы в виде

$$
\sigma_{r 1}=-\frac{2 A b^{2 / n}}{n} I(r)-c_{1} r^{-2 / n}+c_{2},
$$

где $I(r)$ - интеграл от случайной функции

$$
I(r)=\int_{a}^{r} U(x) x^{-1-2 / n} d x
$$

а постоянные интегрирования $c_{1}$ и $c_{2}$ определяются из краевых условий $\sigma_{r 1}(a)=\sigma_{r 1}(b)=0$. В результате решение (17) примет следующий вид: 


$$
\sigma_{r 1}=\frac{2 A b^{2 / n}}{n^{2}}\left(\left(a^{-2 / n}-r^{-2 / n}\right) H-I(r)\right),
$$

где $H=B I(b), B=\frac{1}{a^{-2 / n}-b^{-2 / n}}$.

Далее, подставляя полученные решения (16) и (18) в уравнение (15), имеем

$$
\frac{d}{d r}\left(\sigma_{r 2}^{\prime} r^{1+2 / n}\right)=\frac{2 A b^{2 / n}}{n^{2}}\left[-\frac{2 H U^{\prime}}{n^{2}}+\frac{n+1}{n} U^{\prime} U\right] .
$$

Интегрируя это соотношение, получим решение третьего уравнения системы

$$
\sigma_{r 2}=\frac{2 A b^{2 / n}}{n^{2}}\left[\frac{n+1}{2 n} \int_{a}^{r} U^{2} x^{-1-2 / n} d x-\frac{2 H}{n} I(r)\right]+c_{21}\left(a^{-2 / n}-r^{-2 / n}\right)+c_{22},
$$

где постоянные интегрирования находятся из граничных условий: $\sigma_{r 2}(a)=0, \sigma_{r 2}(b)=0$, откуда следует $c_{22}=0, c_{21}=\frac{2 H^{2}}{n^{2}}-\frac{n+1}{2 n} L$, где $L=B J(b), J(r)=\int_{a}^{r} U^{2}(x) x^{-1-2 / n} d x$.

В результате решение (19) определяется формулой

$$
\sigma_{r 2}=\frac{2 A b^{2 / n}}{n^{2}}\left[\frac{n+1}{2 n} J(r)-\frac{2 H}{n^{2}} I(r)-C\left(r^{-2 / n}-a^{-2 / n}\right)\right],
$$

где введено переобозначение $c_{21}=C$.

Таким образом, получили решение системы, записанное в виде уравнений (16), (18) и (20), которое определяет радиальное напряжение $\sigma_{r}$ во втором приближении.

Теперь найдем приближенные значения компонент тензора скоростей деформаций $\dot{\varepsilon}_{r}$ и $\dot{\varepsilon}_{\varphi}$. Из соотношений (1) и (2) видно, что для этого понадобится величина $\sigma_{\varphi}-\sigma_{r}$, определяемая соотношением (7) и разложением (10). Эту величину с помощью полученных решений (16), (18) и (20) можно представить в виде

$$
\sigma_{\varphi}-\sigma_{r}=r\left(\sigma_{r 0}^{\prime}+\alpha \sigma_{r 1}^{\prime}+\alpha^{2} \sigma_{r 2}^{\prime}\right)=\frac{2 A}{n}\left(\frac{b}{r}\right)^{2 / n}\left[1+\frac{2 \alpha H}{n^{2}}-\frac{\alpha U}{n}-\frac{\alpha^{2} 2 H U}{n^{3}}+\frac{\alpha^{2}(n+1)}{2 n^{2}} U^{2}+\frac{\alpha^{2} 2 C}{n^{2}}\right] .
$$

Возводя последнее соотношение в степень $n$ и подставляя полученное выражение в (1), можно найти выражение для компоненты $\dot{\varepsilon}_{\varphi}$ :

$$
\dot{\varepsilon}_{\varphi}=T\left(\frac{b}{r}\right)^{2}\left[1+\frac{2 \alpha H}{n^{2}}-\frac{\alpha U}{n}-\frac{\alpha^{2} 2 H U}{n^{3}}+\frac{\alpha^{2}(n+1)}{2 n^{2}} U^{2}+\frac{\alpha^{2} 2 C}{n^{2}}\right]^{n}(1+\alpha U),
$$

где $T=\frac{(\sqrt{3})^{n-1} c A^{n}}{n^{n}}$.

Разлагая степенную функцию в ряд Тейлора по $\alpha$ и учитывая только члены до второго порядка малости, получим

$$
\begin{aligned}
& \dot{\varepsilon}_{\varphi}=T\left(\frac{b}{r}\right)^{2}\left[1+\frac{2 \alpha H}{n}-\alpha U-\frac{\alpha^{2} 2 H U}{n^{2}}+\frac{\alpha^{2}(n+1)}{2 n} U^{2}+\frac{\alpha^{2} 2 C}{n}+\right. \\
& \left.+\frac{n(n-1)}{2}\left(\frac{4 \alpha^{2} H^{2}}{n^{4}}-\frac{4 \alpha^{2} H U}{n^{3}}+\frac{\alpha^{2} U^{2}}{n^{2}}\right)+o\left(\alpha^{2}\right)\right](1+\alpha U)= \\
& =T\left(\frac{b}{r}\right)^{2}\left[1+\alpha \frac{2 H}{n}+\frac{2 \alpha^{2}(n+1) H^{2}}{n^{3}}-\frac{\alpha^{2}(n+1) L}{n^{2}}+o\left(\alpha^{2}\right)\right]=-\dot{\varepsilon}_{r} .
\end{aligned}
$$

Найдем основные статистические характеристики случайных скоростей деформаций. Учитывая формулу для интеграла от случайной функции $\langle I(r)\rangle=\int_{a}^{r}\langle U(x)\rangle x^{-1-2 / n} d x$ и замечая, что $<\mathrm{U}(\mathrm{x})>=0$, получаем следующее соотношение для средних скоростей деформаций: 


$$
M\left[\dot{\varepsilon}_{\varphi}\right]=-M\left[\dot{\varepsilon}_{r}\right]=T\left(\frac{b}{r}\right)^{2}\left[1+\frac{2 \alpha^{2}(n+1)\left\langle H^{2}\right\rangle}{n^{3}}-\frac{\alpha^{2}(n+1)\langle L\rangle}{n^{2}}+o\left(\alpha^{2}\right)\right] .
$$

Дисперсии случайных скоростей деформаций вычислялись в предположении, что случайная функция $U(r)$, задающая случайное поле возмущений механических свойств материала, распределена по нормальному закону. В этом случае моменты нечетных порядков равны нулю, а центральные моменты четных порядков выражаются через моменты второго порядка.

Например, центральные моменты четвертого порядка вычисляются по формуле [3]:

$$
\left\langle I_{1}^{0} I_{2}^{0} I_{3}^{0} I_{4}^{0}\right\rangle=k_{12} k_{34}+k_{13} k_{24}+k_{14} k_{23},
$$

где $I_{1}^{0}$ - центрированные случайные величины, $k_{i j}$ - моменты второго порядка.

Рассматривая выражение (21) как сумму зависимых случайных функций, для дисперсий случайных скоростей деформаций можно получить следующее соотношение:

$$
D\left[\dot{\varepsilon}_{\varphi}\right]=T^{2}\left(\frac{b}{r}\right)^{4}\left[\frac{4 \alpha^{4}}{n^{2}} D[H]+\frac{4 \alpha^{4}(n+1)^{2}}{n^{6}} D\left[H^{2}\right]+\frac{\alpha^{4}(n+1)^{2}}{n^{4}} D[L]-\frac{4 \alpha^{4}(n+1)^{2}}{n^{5}}\left\langle\begin{array}{cc}
0 & 0 \\
H^{2} & L
\end{array}\right\rangle\right],
$$

где моменты нечетных порядков опущены.

Моменты второго порядка, входящие в формулу (22) для дисперсий скоростей деформаций, могут быть выражены через корреляционную функцию $K\left(x_{2}-x_{1}\right)$ случайного однородного поля $U(r)$ :

$$
\begin{aligned}
& \left\langle I^{2}(r)\right\rangle=\int_{a}^{r} \int_{a}^{r} K\left(x_{2}-x_{1}\right) x_{1}^{-1-2 / n} x_{2}^{-1-2 / n} d x_{1} d x_{2}, \\
& \langle J(r)\rangle=\int_{a}^{r}\left\langle U^{2}(r)\right\rangle x^{-1-2 / n} d x=\int_{a}^{r} x^{-1-2 / n} d x .
\end{aligned}
$$

Используя формулу (23), а также формулы (25), распишем каждое слагаемое формулы (24):

$$
\begin{gathered}
\left\langle H^{2}\right\rangle=B^{2}\left\langle I^{2}(b)\right\rangle=B^{2} \int_{a}^{b} \int_{a}^{b} K\left(x_{2}-x_{1}\right) x_{1}^{-1-2 / n} x_{2}^{-1-2 / n} d x_{1} d x_{2} ; \\
D\left[H^{2}\right]=\left\langle H^{4}\right\rangle=3\left\langle H^{2}\right\rangle^{2}=3 B^{4}\left(\int_{a}^{b} \int_{a}^{b} K\left(x_{2}-x_{1}\right) x_{1}^{-1-2 / n} x_{2}^{-1-2 / n} d x_{1} d x_{2}\right)^{2} ; \\
D[L]=\left\langle\begin{array}{c}
0 \\
L^{2}
\end{array}\right\rangle=B^{2} \int_{a}^{b} \int_{a}^{b}\left\langle U^{2}\left(x_{1}\right) U^{2}\left(x_{2}\right)\right\rangle x_{1}^{-1-2 / n} x_{2}^{-1-2 / n} d x_{1} d x_{2}= \\
=B^{2}\left(\int_{a}^{b}\left\langle U^{2}\left(x_{1}\right)\right\rangle x_{1}^{-1-2 / n} d x_{1}\right)^{2}+2 B^{2}\left(\int_{a}^{b} \int_{a}^{b}\left\langle U\left(x_{1}\right) U\left(x_{2}\right)\right\rangle x_{1}^{-1-2 / n} x_{2}^{-1-2 / n} d x_{1} d x_{2}\right)^{2}= \\
=B^{2}\left(\int_{a}^{b} x^{-1-2 / n} d x\right)^{2}+2 B^{2}\left(\int_{a}^{b} \int_{a}^{b} K\left(x_{2}-x_{1}\right) x_{1}^{-1-2 / n} x_{2}^{-1-2 / n} d X_{1} d X_{2}\right)^{2}=B^{2}\langle J(b)\rangle^{2}+2 B^{2}\left\langle I^{2}(b)\right\rangle^{2} ; \\
\left\langle H^{2} \prod_{L}^{0}\right\rangle=B^{3} \int_{a}^{b} \int_{a}^{b} \int_{a}^{b}\left\langle U\left(x_{1}\right) U\left(x_{2}\right) U^{2}\left(x_{3}\right)\right\rangle x_{1}^{-1-2 / n} x_{2}^{-1-2 / n} x_{3}^{-1-2 / n} d x_{1} d x_{2} d x_{3}= \\
=B^{3} \int_{a}^{b} \int_{a}^{b} K\left(x_{2}-x_{1}\right) x_{1}^{-1-2 / n} x_{2}^{-1-2 / n} d x_{1} d x_{2} \int_{a}^{b} x^{-1-2 / n} d x+2 B^{3}\left(\int_{a}^{b} \int_{a}^{b} K\left(x_{2}-x_{1}\right) x_{1}^{-1-2 / n} x_{2}^{-1-2 / n} d x_{1} d x_{2}\right)^{2}= \\
=\left\langle H^{2}\right\rangle B\langle J(b)\rangle+2 B^{3}\left\langle I^{2}(b)\right\rangle^{2} .
\end{gathered}
$$

Статистическая обработка опытных данных показывает, что корреляционные функции механических характеристик являются знакопеременными затухающими функциями $[4,5]$, и их можно аппроксимировать выражением:

или более сложным соотношением

$$
K(\rho)=e^{-\gamma|\rho|} \cos (\beta \rho), \quad \rho=x_{2}-x_{1}, \quad \gamma>0
$$




$$
K(\rho)=e^{-\gamma|\rho|}\left(\cos (\beta \rho)+\frac{\gamma}{\beta} \sin \beta|\rho|\right),
$$

где $\gamma, \beta$-постоянные величины, определяемые по опытным данным из условий наилучшей аппроксимации.

Случайное поле микронеоднородностей, корреляционная функция которого задается выражением (26), не является дифференцируемым, что приводит к затруднениям при оценке надежности элементов конструкций по теории выбросов. Случайное поле с законом корреляции (27) дифференцируемо. Поэтому численные вычисления моментов второго порядка (25) проведены в предположении, что корреляционная функция случайного однородного и одномерного поля неоднородностей $U(r)$ имеет вид (27):

$$
K(\rho)=e^{-10|\rho|}\left(\cos (20 \rho)+\frac{1}{2} \sin 20|\rho|\right) .
$$

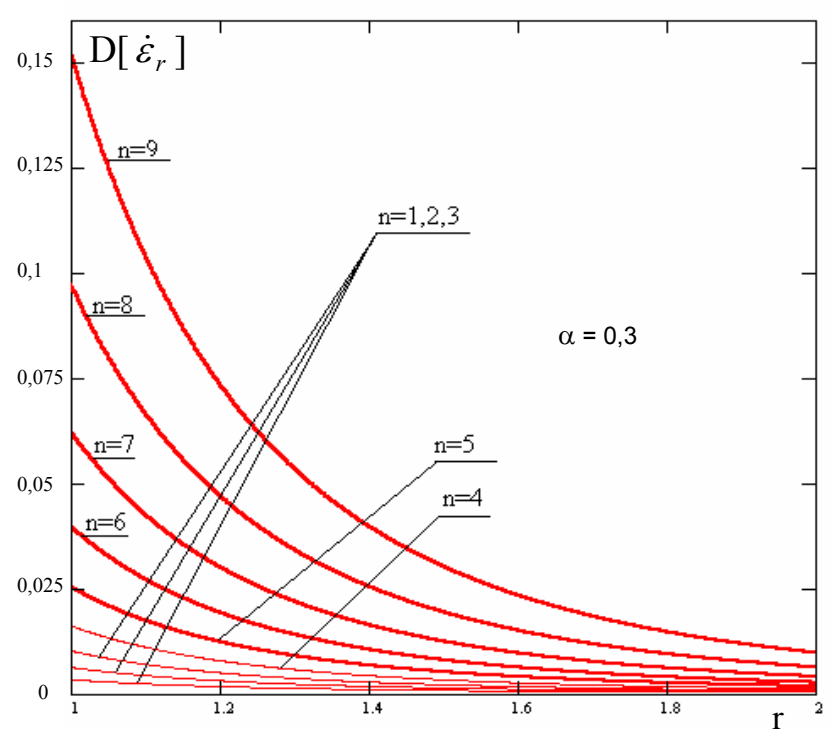

Р и с. 1. Графики дисперсий скоростей деформаций в зависимости от $r$ при различных $n$

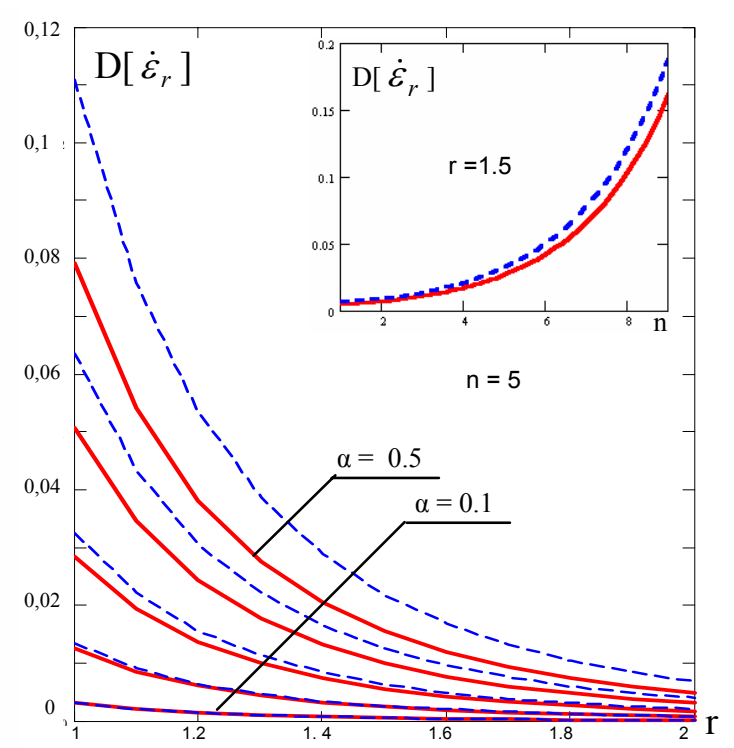

Р и с. 2. Графики дисперсий скоростей деформаций в первом (сплошные линии) и втором (пунктирные линии) приближениях при различных $\alpha$ и $n$

Дисперсии скоростей деформаций в первом и втором приближениях при различных $\mathrm{n}$ и $\alpha$

\begin{tabular}{|c|c|c|c|c|c|c|c|}
\hline & $\alpha \backslash n$ & 1 & 3 & 5 & 7 & 9 & 11 \\
\hline D1 & & 0.0005 & 0.0012 & 0.0030 & 0.0073 & 0.0180 & 0.0441 \\
\hline D2 & 0,1 & 0.0005 & 0.0013 & 0.0031 & 0.0075 & 0.0181 & 0.0443 \\
\hline D1 & & 0.0023 & 0.0050 & 0.0120 & 0.0290 & 0.0720 & 0.1750 \\
\hline D2 & 0,2 & 0.0026 & 0.0055 & 0.0130 & 0.0320 & 0.0770 & 0.1870 \\
\hline D1 & & 0.0051 & 0.0110 & 0.0270 & 0.0660 & 0.1610 & 0.3930 \\
\hline D2 & 0,3 & 0.0071 & 0.0140 & 0.0330 & 0.0780 & 0.1890 & 0.4580 \\
\hline D1 & & 0.0090 & 0.0200 & 0.0480 & 0.1180 & 0.2870 & 0.6990 \\
\hline D2 & 0,4 & 0.0160 & 0.0280 & 0.0650 & 0.1550 & 0.3730 & 0.9030 \\
\hline D1 & & 0.0140 & 0.0310 & 0.0760 & 0.1840 & 0.4480 & 1.0920 \\
\hline D2 & 0,5 & 0.0300 & 0.0510 & 0.1160 & 0.2750 & 0.6590 & 1.5890 \\
\hline
\end{tabular}

Численные вычисления по формуле (24) для толстостенной трубы с внутренним и наружным радиусами соответственно - $a=1, b=2$, показали, что дисперсии скоростей деформаций $\mathrm{D}\left[\dot{\varepsilon}_{r}\right]$ и $\mathrm{D}\left[\dot{\varepsilon}_{\varphi}\right]$ с увеличением $n$ увеличиваются, причем наибольшие дисперсии вблизи внутренней поверхности трубы, а наименьшие - в окрестности наружной поверхности трубы. Данное утверждение проиллюстрировано графиками дисперсий как функций, зависящих от $r$, изображенных на рис.1. На рис.2 изображен график, показывающий различия дисперсий, посчи- 
танных в первом (сплошная линия) и втором (пунктир) приближениях. В правом верхнем углу рис.2 приведен срез этого графика при $r=1.5$, показывающий зависимость дисперсий от показателя нелинейности $n$.

В таблице представлены значения дисперсий скоростей деформаций в зависимости от $n$ и $\alpha$ при $r=1,5:$ в первой строке приведены значения, вычисленные по первому приближению; во второй - по второму приближению.

Из приведенных графиков и таблицы видно, что для слабонеоднородных материалов $(\alpha=0.1-0.2)$ значения дисперсий скоростей деформаций отличаются незначительно. Для материалов с большой степенью неоднородности $(\alpha=0.4-0.5)$ значения дисперсий скоростей деформаций, вычисленные по второму приближению, могут превосходить соответствующие значения, вычисленные по первому приближению, в полтора раза. Поэтому в данном случае неучет членов второго порядка малости может привести к необоснованному завышению показателей прочности и надежности толстостенной трубы.

\section{БИБЛИОГРАФИЧЕСКИЙ СПИСОК}

1. Попов Н.Н., Самарин Ю.П. Пространственная задача стационарной ползучести стохастически неоднородной среды // ПМТФ. 1985. №2. С. 150-155.

2. Качанов Л.М. Теория ползучести. М.: Физматгиз, 1960. 455 с.

3. Свешников А.А. Прикладные методы теории случайных функций. М.: Наука, 1968. 464 с.

4. Кукса Л.В., Лебедев А.А., Ковальчук Б.И. О законах распределения микродеформаций в двухфазных поликристаллических сплавах при простом и сложном нагружениях // Проблемы прочности. 1986. №1. С. 7-11.

5. Богачев И.И., Вайнштейн А.А., Волков С.Д. Статистическое металловедение. М.: Металлургия, 1984.176 с. 